\title{
Estudo e Aplicação da Tecnologia de Redes de Acesso Genérico ao Núcleo da Rede GSM
}

\author{
Gleidson G. C. B. do Nascimento ${ }^{1}$, Renato M. de Moraes $^{2}$
}

\begin{abstract}
Resumo - Este artigo revisa e aplica a tecnologia de redes de acesso genérico (GAN) ao núcleo do sistema global para comunicação móvel (GSM) apresentando uma análise dos recursos oferecidos e necessários para sua aplicação. Sua finalidade é oferecer uma alternativa de obter acesso ao núcleo da rede GSM aos usuários fora da área de cobertura de sua operadora, porém mantendo uma qualidade de serviço satisfatória. Realizamos um estudo de caso sobre a aplicação desta tecnologia, indicando suas vantagens e desvantagens através de comparações simples em serviços básicos, como tempo de registro, tempo de chamada e custos, para melhor entender esta tecnologia alternativa que integra múltiplos meios de acesso distintos.
\end{abstract}

Palavras-Chave - GSM, redes de acesso genérico (GAN), redes de comunicação, sistemas celulares, UMTS.

Abstract - This paper reviews and applies the generic access network (GAN) technology to the core of the global system for mobile communication (GSM) in which we present analysis of the available and necessary resources for its application. The GAN goal is to provide an alternative to the system users, who are outside their coverage area, with satisfactory quality of service. We perform a case study for application of this technology, indicating its advantages and disadvantages through simple comparisons in basic services, as registry time, call time and costs in order to better understand this alternative technology which integrates multiples and distinct means of access.

Keywords - Communication systems, generic access networks (GAN), GSM, radio cellular systems, UMTS.

\section{INTRODUÇÃO}

O mercado de comunicações móveis está passando por profundas mudanças e adquirindo características bem específicas, como o aumento da penetração do sinal, da taxa de transferência da rede, da quantidade de serviços disponibilizados aos clientes, aliado as outras características de mercado, como a fácil mutação de objetivos, à procura pelo aumento das margens de lucro, entre outros. Uma das soluções para agregar grande parte destes fatores é a busca por novas tecnologias que promovam a convergência de rede e de serviços, que juntamente com uma redução significativa de custos, atrairão mais os clientes [1].

Segundo informações da GSM Association [2], estima-se que aproximadamente três bilhões de pessoas utilizaram o sistema GSM em suas linhas de comunicação móvel até o final de 2007. Isto se deve ao aumento constante da cobertura do sinal em todo o globo, além do desenvolvimento da infraestrutura encontrada por trás do sistema. Para o usuário final,

\footnotetext{
${ }^{1}$ Departamento de Engenharia Elétrica, Escola Politécnica, Universidade de Pernambuco (UPE) e Centro de Estudos Avançados do Recife (CESAR).

2 Departamento de Sistemas e Computação, Escola Politécnica, Universidade de Pernambuco (UPE).

E-mails: gleidson.correia@gmail.com, renato@dsc.upe.br.
}

estes números são sinônimos de um sistema eficaz, robusto e pouco falho. Para a operadora, é o fruto de altos investimentos desprendidos para garantir a fidelidade de seus usuários aliada a um custo reduzido [3].

Uma das opções disponíveis que visa este tipo de investimento é a tecnologia de redes de acesso genérico (GAN) ao núcleo da rede GSM, que visa expandir ainda mais a arquitetura de rede GSM através de novas formas de acesso, como a Internet, às redes de área locais (Local Area Network, LAN), às redes locais sem fio (Wireless Local Area Networks, WLAN), entre outras redes particulares sem contato com a rede do sistema GSM. No entanto, esta implementação precisa ser vantajosa para a operadora e seus clientes. A tecnologia de acesso genérico ao núcleo da rede GSM foi desenvolvida pelo 3GPP (Third Generation Partneship) para suprir algumas limitações na rede GSM e na Universal Mobile Telecommunications (UMTS) esta última uma evolução direta do GSM, como a ausência de cobertura de sinal, excesso de custo ao utilizar redes pertencentes a outras operadoras e segurança relativamente baixa no espectro [4]. Diante do exposto, este artigo busca descrever o funcionamento da tecnologia GAN, suas características e como ela se encaixa nas tecnologias celulares e IP existentes. Realizaremos um estudo de caso onde iremos comparar os tempos médios de registro e de realização de chamadas num sistema padrão GSM e usando o GAN, ressaltando o método alternativo de acesso ao núcleo da rede nos casos em que o usuário encontra-se fora da área de cobertura de sua operadora.

O restante deste trabalho está organizado da seguinte forma. Na seção II explicamos as redes GSM e UMTS. Na seção III descrevemos o acesso genérico a estas duas redes, assim como sua implantação física e lógica. Na seção IV comparamos o acesso comum e o genérico, com os devidos comentários, vantagens e desvantagens da tecnologia, utilizando uma pesquisa exploratória, em campo, para obter dados relevantes sobre a tecnologia, como os tempos de operação e custos. Por fim, na seção $V$ expomos as conclusões e sugestões de trabalhos futuros.

\section{A REDE GSM E UMTS}

O sistema global para comunicação móvel (Global System for Mobile Communication, GSM) é o nome de um grupo de padronização de sistemas de comunicação celular digital formado em 1982 com a intenção de criar um sistema de telefonia móvel comum para a Europa. O GSM possui um sistema poderoso de sinalização que facilita o funcionamento do aparelho celular fora de sua localidade padrão (roaming), através de detecção e registro de localização na rede automaticamente. Também provê mobilidade ao usuário final, com a criação do Módulo de Identidade do Usuário, o SIM (Subscriber Identity Module), que possui o número definido pela operadora ao mesmo [4], [5]. 
No início do século XXI, o mesmo grupo criador do sistema GSM criou os estágios iniciais do primeiro sistema de comunicações da terceira geração, o UMTS. A primeira rede UMTS foi utilizada com sucesso na Ilha de Man, dentro das ilhas britânicas, pela empresa O2. O UMTS utiliza um novo padrão de interface aérea, chamado de Acesso Múltiplo por Divisão de Código em Banda Larga, WCDMA (Wide-Band Code-Divison Multiple Access), adaptado de um padrão japonês chamado FOMA (Freedom of Mobile Access) [6], [7]. Este sistema permite altas taxas de transmissão de dados, na ordem de 2 Mbits por segundo, além de um alcance e pervasividade maior que o padrão GSM.

O sistema UMTS é uma evolução direta do GSM. Os usuários deste sistema não terão diferença em sua cobertura caso estejam em uma área apenas provida pelo sistema UMTS. Por sua vez, um aparelho UMTS terá apenas os serviços GSM em uma situação inversa a anterior. A estrutura de rede do sistema, assim como o GSM, é transparente para o usuário final e dividida em duas partes: o subsistema de rede de acesso de radio ou RAN (Radio Access Network); o núcleo de rede ou CN (Core Network), parte responsável pela interconexão de redes e controle de usuários, herdando as funções da rede GSM e agregando novas funções. Os elementos principais do núcleo do sistema são [4], [5]: Mobile Switching Center (MSC) é o local onde as ligações são interconectadas; Home Location Register (HLR) é um banco de dados dos usuários; Authentication Centre (AuC) é o local onde o SIM é autenticado; Visitor Location Register (VLR) é um banco de dados temporário para usuários em roaming; Equipment Identity Register (EIR) lista de aparelhos banidos do sistema ou sob monitoração; Serving GPRS Support Node (SGSN) é a parte responsável pela entrega de pacotes GPRS aos aparelhos da rede; Gateway GPRS Support Node (GGSN) é a interface entre GPRS e outras redes tais como a Internet. Estas tecnologias possuem algumas limitações. Listaremos aqui as que foram relevantes para a produção deste artigo:

- Limite de cobertura: apesar de o GSM ser uma tecnologia mundialmente aceita e encontrada em mais da metade do globo terrestre, nem todos os lugares possuem antenas irradiando sinal GSM, e é extremamente necessário cobertura para que os serviços estejam disponíveis ao usuário [4], [6];

- Demanda de tráfego: como em qualquer outra tecnologia, a alta demanda associada a pouca oferta resulta em baixa qualidade de serviço oferecido. O aumento considerável da utilização da tecnologia leva ao estrangulamento da banda, impedindo a operadora de ofertar mais qualidade a seus serviços [7], [8];

- Custo elevado: a utilização da rede em algumas situações pode levar a um gasto muito alto para o usuário, tornando a utilização dos serviços extremamente dispendiosa em situações de relativa necessidade. Basicamente, um celular está filiado a sua rede de origem. Quando se desloca para um lugar fora de sua rede de origem, embora ainda na cobertura da operadora, paga-se um preço um pouco mais caro. Se utilizar a rede de outra operadora, o preço a pagar será bem maior [3], [5], [7];

- Necessidade de convergência: a rápida evolução da tecnologia da informação e da telefonia celular levou a uma busca desenfreada por serviços que agreguem mais valor ao usuário, a custos menores. A busca por serviços assim são norteadas pelo mercado, pela necessidade de oferta e pela atual demanda, e a tecnologia GSM é deficitária neste perfil, por possuir baixas taxas de transferência de dados [9]-[11].

\section{O ACESSO GENÉRICO}

Com as limitações listadas na seção anterior, podemos concluir que um celular fora da cobertura de sua operadora está sujeito às tarifas extras aplicadas tanto pela operadora do cliente como pelas outras operadoras que ele precise utilizar. Além disto, o usuário pode ficar limitado apenas aos serviços oferecidos por esta outra operadora. Como o aparelho celular é um equipamento que utiliza redes sem fio, a utilização de redes alternativas (desde que suportado pelo hardware do aparelho) seria uma possível solução para este problema. Mesmo assim, ainda haveria a questão de acessar o núcleo da rede GSM. Porém, este acesso poderia ser feito através da Internet, se o núcleo da rede suportasse formas alternativas de acesso como o acesso IP pela Internet. No sistema GSM, os equipamentos são acessíveis através de suas respectivas interfaces. Sendo assim, para termos acesso ao núcleo da rede, as seguintes interfaces precisariam estar disponíveis: a interface A da MSC para voz e a interface Gb da SGSN para dados [12], [13].

O núcleo da rede posiciona-se desfavoravelmente dentro da rede interna da operadora. Por sua vez, a Internet é uma rede multi-protocolos, os quais não são diretamente compatíveis com os padrões do sistema GSM/UMTS. Logo, seria inviável (e complicado) expor diretamente estas interfaces para a Internet. Portanto, é necessário um aparelho que possa intermediar as transferências entre a Internet e as interfaces, mantendo a segurança oferecida pelo sistema e a interconectividade disponível. Foi com este pensamento que a 3GPP desenvolveu em 2005 as especificações TS 43.318 e 44.318 para regulamentar o acesso genérico às interfaces A e Gb, ou redes de acesso genérico (Generic Access Networks, GAN) [12], [13], cuja função é receber da Internet as informações de aparelhos devidamente registrados na operadora e convertê-los ao padrão de sinalização referente ao serviço utilizado no momento. A Figura 1 ilustra as duas possibilidades de acesso à operadora: o acesso padrão (ou comum) local através da estação rádio base (Base Transceiver System, BTS), ou através de um ponto de acesso (AP) sem fio (WiFi) no caso em que a BTS não esteja disponível [12], [13].

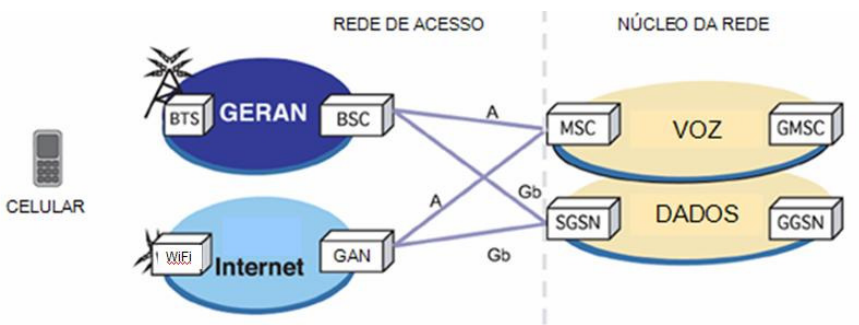

Fig. 1. Visão geral do acesso genérico (GAN) e do acesso padrão (GSM).

Devido à conexão permanente com a Internet, as interfaces A e $\mathrm{Gb}$ poderão servir a este celular como se ele estivesse em sua cobertura GSM padrão. Isto significa dizer que todos os serviços disponíveis para o celular na cobertura padrão devem por obrigação estar disponíveis também no acesso genérico. $\mathrm{O}$ acesso genérico ao núcleo da rede celular é feito pela Internet utilizando o protocolo de tempo real (RTP), usando-se o protocolo de datagramas de usuário (UDP) em portas acima de 1024 [9], [11]. 


\section{A. Implantação Física e Lógica do Acesso Genérico}

Faremos agora um estudo de caso de implantação da GAN em uma operadora GSM genérica e sua utilização pelo usuário, através de um aparelho celular (ou Mobile System, MS) compatível com a tecnologia. Como citado na seção anterior, as interfaces A (para voz) e Gb (para dados) serão acessadas através do aceso genérico pelo celular, mas no estudo de caso iremos considerar apenas o acesso à interface A. $\mathrm{O}$ acesso à interface $\mathrm{Gb}$ é feito de maneira semelhante seguindo a camada de protocolos de dados do núcleo, mas não será mencionado devido ao fato de que o celular também é capaz de alcançar a Internet e solicitar serviços pelo ponto de acesso.

A adaptação, por parte da operadora genérica, requer a utilização dos seguintes elementos de rede:

- Generic Access Network Controller (GANC): serve para transportar e converter os dados entre o núcleo da operadora (acessível via a interface A da MSC) e o celular (acessível via Internet);

- Security Gateway (SEGW): elemento responsável por estabelecer um túnel seguro IPSec entre a rede e o celular;

- Authentication, Authorization and Accounting (AAA): elemento responsável por prover autenticação a qualquer instante para o GANC e o SEGW enquanto o celular não tem acesso ao núcleo da rede;

- Network Management System (NMS): elemento responsável pela gerência do sistema como um todo, atuando como cãode-guarda, obtendo registros e relatórios de desempenho e informando o centro de gerência de redes 24 horas por dia;

- Load Balancing Router (LBR): balanceia a carga demandada pelos celulares e também pela operadora, garantindo qualidade de serviço de voz e dados (Quality of Service, $Q o S)$;

- Media Gateway (MGW): elemento atuante como tradutor entre sistemas de comunicação diferentes. No caso aqui tratado, é responsável por conectar a MSC ao sistema GAN.

Estes elementos são instalados na central de dados (datacenter) da operadora e estão na mesma rede local dos outros aparelhos que a operadora já possui. Além disto, as seguintes ações são necessárias: aquisição de um enlace com a Internet, com endereço IP válido, e obtenção de um nome de domínio completo (Fully Qualified Domain Name, FQDN) associado ao endereço IP válido. A Figura 2 mostra como os elementos acima descritos estão conectados.

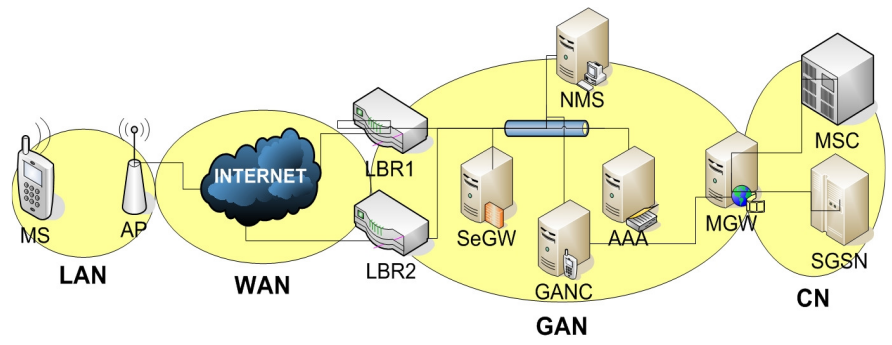

Fig. 2. Projeto do acesso genérico.

$\mathrm{Na}$ prática, todo o equipamento fica montado em um bastidor, em três dimensões, medindo $2,20 \mathrm{~m}$ de altura, $0,48 \mathrm{~m}$ de comprimento e $0,70 \mathrm{~m}$ de profundidade. Dentro dele, estão presentes o GANC, os dois LBR's, o SEGW, AAA, NMS e MGW, como também estão instalados uma fonte de energia de $24 \mathrm{~V}$ e um dissipador de calor, ambos no topo.
Dado que o acesso genérico ao núcleo da rede celular é feito pela Internet, sua camada de protocolos mescla camadas pertencentes à Internet (modelo TCP/IP) e a rede GSM (sem a camada de interface de rádio aérea), como ilustra a Figura 3 para acesso à interface $\mathrm{A}$.

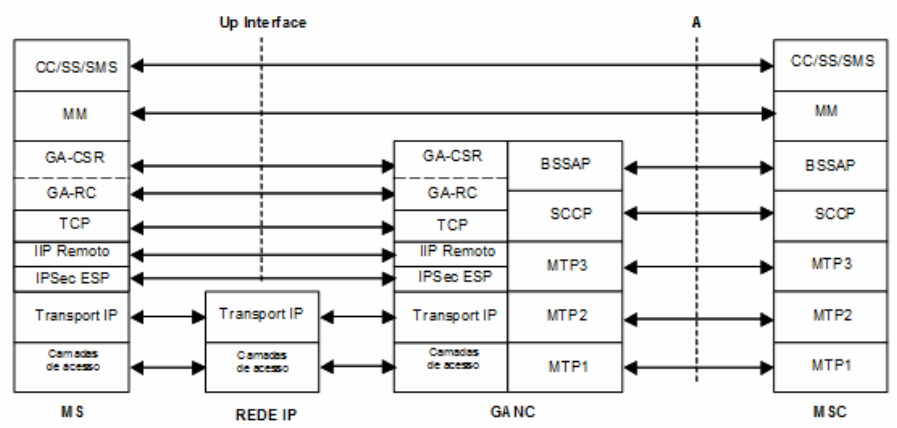

Fig. 3. Camadas de protocolo do acesso genérico para serviços de voz.

Observe que, na Figura 3, há uma linha tracejada entre o celular e a rede IP, que representa a Internet, e aqui neste trabalho também será considerada uma interface, chamada Up. Na interface Up, as camadas de baixo da rede IP, do celular e da rede são responsáveis por prover a conectividade de forma genérica. Pelo fato de a Internet ser uma rede ampla e consolidada, o acesso genérico está habilitado a receber e tratar dados provenientes de qualquer parte do globo [13]. No entanto, o processo de passagem da Internet para o GSM não é tão simples e automático, pois existem alguns problemas técnicos e operacionais, a saber:

- Segurança: neste caso, o acesso genérico está sendo feito pela Internet, uma rede pública de livre acesso, logo, sujeita a vários riscos como captura do conteúdo das mensagens trocadas entre o celular e a operadora, vulnerabilidade a pessoas ou códigos maliciosos que podem causar perda da integridade de suas informações, perda da acessibilidade de um dos elementos, entre outros problemas em potencial;

- Autenticação: tanto a operadora quanto o celular precisam se certificar de que a comunicação é realizada entre eles e não por um elemento estranho tentando se passar por algum dos dois;

- Gerência: mesmo estando fora da área de cobertura normal, o celular necessita do envio e recebimento das mesmas informações que um outro celular localizado em cobertura GSM. Por isso, a estrutura de gerência da operadora deve contemplar esta nova situação do aparelho.

O acesso genérico foi possível devido ao acréscimo de novos elementos que visaram garantir a integridade dos dados, a privacidade das informações trocadas e a autenticidade de ambas as partes. Isto acrescentou novos processos na máquina de estados do celular, que afetam desde o registro, um procedimento simples e constante, até uma chamada de voz e transferência de dados. Na Figura 4 podemos observar a interação da segurança entre as camadas de acesso genérico.

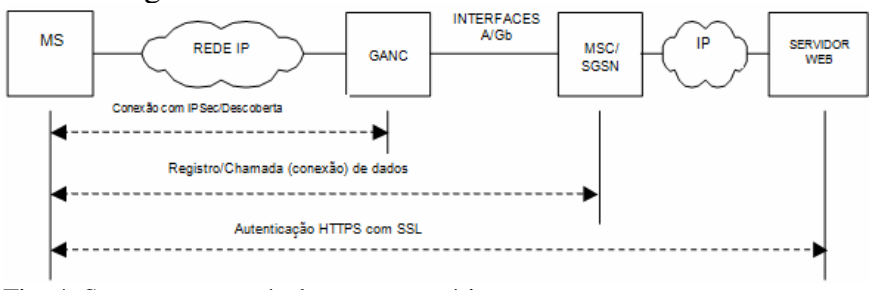

Fig. 4. Segurança através do acesso genérico. 

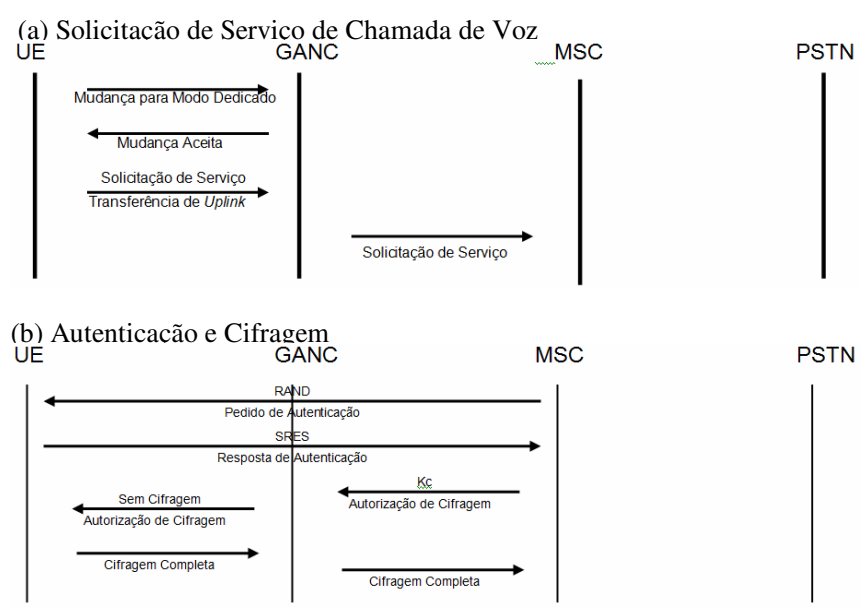

PSTN
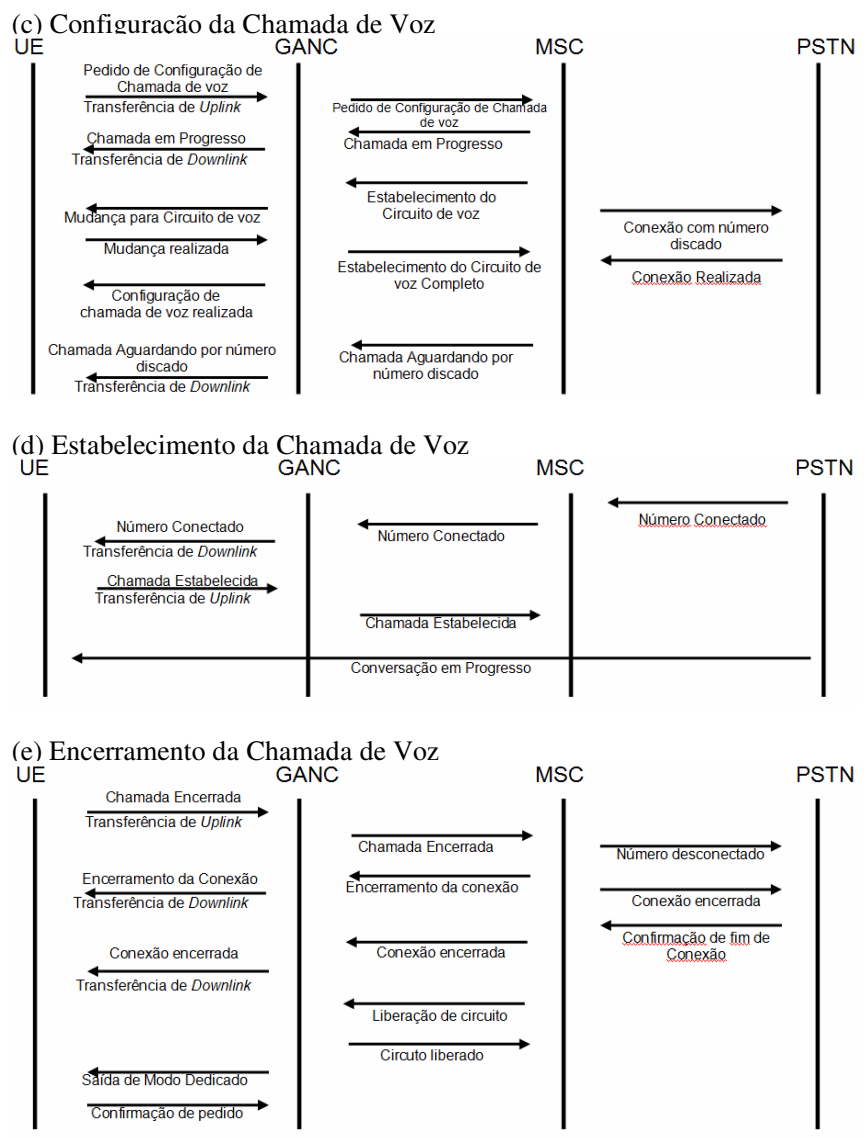

Fig. 5. Exemplo de uma chamada de voz para o acesso genérico.

A comunicação entre o aparelho e a rede sofreu algumas alterações no acesso genérico, referentes à sinalização realizada durante a solicitação de serviços, entre outros. Os sistemas GSM e UMTS funcionam de forma semelhante. Além de utilizarem a mesma estrutura de rede, possuem as mesmas interfaces de acesso. De fato, há uma diferença grande nas camadas de protocolo de sinalização, porém os dois sistemas acessam as interfaces da MSC de forma similar, utilizando as interfaces A e $\mathrm{Gb}$. $\mathrm{O}$ acesso genérico atua, neste caso, como um tradutor de comandos, passando a sinalização recebida pelo núcleo da rede de forma que o celular compreenda e da mesma forma devolve o comando recebido do celular em um formato compatível para a MSC. Utilizando uma chamada de voz como exemplo, mostrado na Figura 5, percebemos que ela será realizada apenas quando o celular estiver devidamente registrado no acesso genérico e o túnel seguro IPSec estiver estabelecido. Neste exemplo, consideramos que o celular está em estado inativo, isto é, sem nenhuma atividade dedicada de rede no momento. Sendo assim, uma chamada de voz se dá conforme descrito na Figura 5, onde as fases (a) a (e) acontecem [12], [13].

\section{COMPARAÇÃO ENTRE O ACESSO GENÉRICO E O COMUM}

Nesta seção, realizamos uma comparação do tempo gasto pelo celular utilizando as duas formas de acesso, analisando os resultados obtidos. Para tal, empregamos o sistema de uma operadora real, a americana AT\&T. Além disto, também utilizamos a estrutura da rede GSM T-Mobile, localizada naquele mesmo país. Foram utilizados dois aparelhos celulares, de mesmo modelo (A910) e fabricante (Motorola), com suporte a WiFi e GAN, dois USIMs, sendo um pertencente à T-Mobile, na rede dos Estados Unidos, mas registrado na rede da TIM em roaming, e o outro pertencente à AT\&T, na rede americana, registrado via GAN.

Os testes consistiram em verificar as diferenças de tempo entre o acesso pela GAN e pela rede GSM. O objetivo destes testes foi mostrar a viabilidade do sistema, provar que o tempo de acesso ou a diferença entre a forma da utilização do sistema, mesmo existente, é relativamente pequena para impactar a sua utilização, e explicar as vantagens e desvantagens da utilização deste sistema juntamente com o sistema GSM. Também fizemos uma simples comparação entre os preços aplicados nas duas formas de acesso.

Primeiramente, foi medido o tempo de registro, separadamente, nas duas redes. $\mathrm{O}$ instante inicial para este teste é o instante em que o celular é ligado. Consideramos o celular registrado quando ele informa o nome da operadora em sua tela, juntamente com a mensagem de inundação de célula (cell broadcast) recebida com a confirmação de registro. Este procedimento foi repetido 100 vezes para cada rede e os tempos encontrados estão ilustrados na Figura 6.

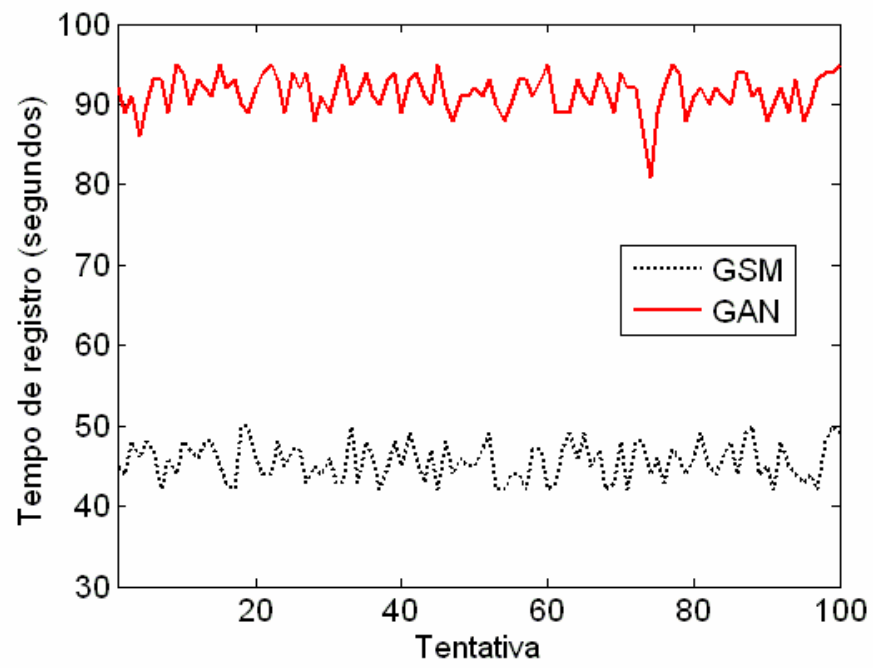

Fig. 6. Tempo de registro para o acesso comum (GSM) e o genérico (GAN).

A Tabela I mostra as médias e variâncias referentes às medidas da Figura 6. 
TABELA I

Média e variância dos tempos de registro referentes à Figura 6 .

\begin{tabular}{|c||c|c|}
\hline Rede & Média (segundos) & Desvio Padrão (segundos) \\
\hline \hline GSM & 45,6 & 2,3 \\
\hline GAN & 91,4 & 2,4 \\
\hline
\end{tabular}

Com relação ao tempo de registro, percebemos que de fato, há um tempo maior gasto pelo celular no processo de registro quando a tecnologia GAN é usada. Este tempo maior se deve à inicialização do serviço, que é a localização e conexão com um AP conhecido, busca de DNS pelo FQDN do SEGW, e a autenticação e estabelecimento de um túnel seguro IPSec com o SEGW. Dependendo das condições da rede e do sinal WiFi, este tempo pode aumentar ainda mais. Com relação ao sistema GAN, há um número maior de autenticações e verificações realizadas pelo aparelho e pela operadora, devido ao modelo de segurança, que claramente é mais vantajoso em relação ao modelo do sistema GSM. Considerando o fato de que o registro ocorre apenas quando o celular é ligado, e é mantido pelo aparelho enquanto ele estiver ligado, o tempo de registro, apesar de grande, não é um empecilho direto ao uso da tecnologia, mas é uma desvantagem em relação ao sistema GSM. Além disso, o baixo desvio padrão encontrado nas duas redes mostra a estabilidade no processo de registro da rede genérica e na comum. Outra vantagem é a possibilidade de registrar na rede o seu aparelho em qualquer lugar onde não há cobertura GSM, mas exista $\mathrm{WiFi}$, o que pode levar o alcance de uma operadora a praticamente todo o globo.

Outra consideração importante é que o sistema GAN informa ao aparelho sua localidade, relativa ao ponto de acesso genérico onde ele se registrou. Esta informação é vantajosa para o usuário e para a operadora porque alguns celulares têm em seu software um algoritmo que utiliza este parâmetro para decidir se é mais barato realizar a ligação utilizando a rede genérica ou o sistema comum GSM.

Em seguida, medimos separadamente o tempo gasto pelo celular (e a rede) para realizar uma chamada de voz a um número de destino. $\mathrm{O}$ instante inicial para a contagem do tempo é quando o usuário pressiona o botão enviar (send) para iniciar a chamada, enquanto que o instante final é quando o número de destino começa a chamar. Este procedimento foi repetido 100 vezes para cada rede e os tempos encontrados estão ilustrados na Figura 7.

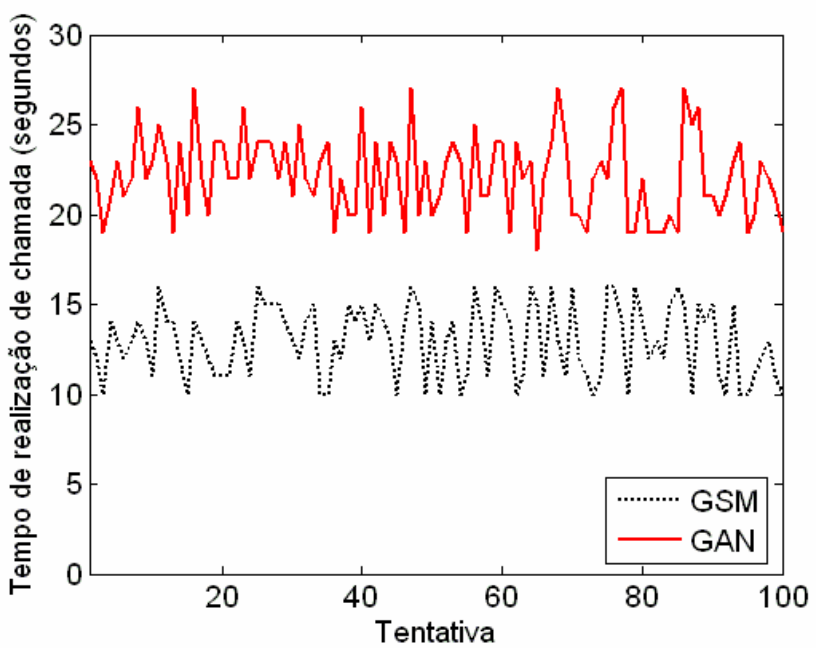

Fig. 7. Tempo de realização de chamada de voz para o acesso comum (GSM) e o genérico (GAN).
A Tabela II mostra as médias e variâncias referentes às medidas da Figura 7.

TABELA II

Média e variância dos tempos de realização de chamada de voz referentes à Figura 7.

\begin{tabular}{|l||c|c|}
\hline Rede & Média (segundos) & Desvio Padrão (segundos) \\
\hline \hline GSM & 12,9 & 2,0 \\
\hline GAN & 22,2 & 2,3 \\
\hline
\end{tabular}

Com relação ao tempo de realização de chamadas de voz, observe que o celular não possui quedas significativas no tempo de utilização do serviço no sistema GSM (ilustrado pela baixa diferença entre os desvios padrão), mas, de fato, é mais variável no sistema GAN, devido à instabilidade da rede, excesso de tráfego no meio, ruídos ou interferência na interface aérea ou ainda alta latência na Internet. Mesmo assim, o sistema realizou todas as chamadas discadas, e também conseguiu uma diferença de tempo pequena em relação ao teste de registro, em uma média de oito segundos de diferença. Na prática, o curto tempo de realização de uma chamada no sistema GSM é mantido no sistema GAN com uma diferença de poucos segundos a mais, mostrando que o acesso genérico é uma alternativa confiável ao GSM, sem retirar a qualidade ou a velocidade de acesso aos recursos localizados no núcleo da rede. Além disto, o curto tempo de espera entre as redes para a realização da chamada de voz reduz a diferença encontrada entre as mesmas.

A Tabela III mostra os custos de alguns serviços no acesso padrão (GSM). Esta tabela foi montada considerando uma média aritmética de três operadoras distintas que realizam todos os serviços indicados da mesma maneira. $\mathrm{O}$ custo pode variar, apenas estamos ressaltando do ponto de vista da operadora, quais tipos de custos seriam arcados pelo usuário final na utilização de cada tipo de serviço. A unidade é centavos de dólar por minuto, e msg significa mensagem.

TABELA III

Custos no acesso padrão GSM.

\begin{tabular}{|c|c|c|c|c|}
\hline \multirow[b]{2}{*}{ Serviço } & \multicolumn{4}{|c|}{ Destino } \\
\hline & $\begin{array}{l}\text { Mesma rede e } \\
\text { localidade }\end{array}$ & $\begin{array}{c}\text { Mesma rede e } \\
\text { outro lugar }\end{array}$ & $\begin{array}{l}\text { Outra rede e } \\
\text { mesmo lugar }\end{array}$ & $\begin{array}{l}\text { Outra rede e } \\
\text { localidade* }\end{array}$ \\
\hline $\begin{array}{l}\text { Chamada } \\
\text { de Voz }\end{array}$ & $\begin{array}{c}(0,15+0,10 \\
+0,15) / 3= \\
\$ 0,13 / \mathrm{min} \\
\end{array}$ & $\begin{array}{c}\$(0,25+0,20 \\
+0,25) / 3= \\
\$ 0,20 / \mathrm{min} \\
\end{array}$ & $\begin{array}{c}(0,18+0,15 \\
+0,20) / 3= \\
\$ 0,18 / \mathrm{min} \\
\end{array}$ & $\begin{array}{c}(0,25+0,20 \\
+0,25) / 3= \\
\$ 0,20 / \mathrm{min} \\
\end{array}$ \\
\hline $\begin{array}{l}\text { Chamada } \\
\text { de Dados }\end{array}$ & $\begin{array}{c}\$(4,99+19,99 \\
+9,99) / 3= \\
\$ 11,65 / \mathrm{MB}\end{array}$ & $\begin{array}{c}\$(4,99+19,99 \\
+\$ 9,99) / 3= \\
\$ 11,65 / \mathrm{MB}\end{array}$ & $\begin{array}{c}\$(4,99+19,99 \\
+9,99) / 3= \\
\$ 11,65 / \mathrm{MB}\end{array}$ & $\begin{array}{c}\$(4,99+19,99 \\
+9,99) / 3= \\
\$ 11,65 / \mathrm{MB}\end{array}$ \\
\hline $\begin{array}{l}\text { SMS } \\
\text { (torpedos) }\end{array}$ & $\begin{array}{c}\$(0,30+0,30 \\
+0,30) / 3= \\
\$ 0,30 / \mathrm{msg}\end{array}$ & $\begin{array}{c}\$(0,30+0,30 \\
+0,30) / 3= \\
\$ 0,30 / \mathrm{msg}\end{array}$ & $\begin{array}{c}\$(0,30+0,30 \\
+0,30) / 3= \\
\$ 0,30 / \mathrm{msg}\end{array}$ & $\begin{array}{c}\$(0,30+0,30 \\
+0,30) / 3= \\
\$ 0,30 / \mathrm{msg}\end{array}$ \\
\hline
\end{tabular}

A Tabela IV mostra os custos dos mesmos serviços da tabela anterior para o acesso genérico (GAN). A unidade é centavos de dólar por minuto, e msg significa mensagem.

TABELA IV

Custos no acesso genérico (GAN).

\begin{tabular}{|l||c|c|c|c|}
\hline \multicolumn{1}{|c||}{ Serviço } & $\begin{array}{c}\text { Mesma rede, } \\
\text { mesma } \\
\text { localidade }\end{array}$ & $\begin{array}{c}\text { Mesma rede, } \\
\text { outra } \\
\text { localidade }\end{array}$ & $\begin{array}{c}\text { Outra rede, } \\
\text { mesma } \\
\text { localidade }\end{array}$ & $\begin{array}{c}\text { Outra rede, } \\
\text { outra } \\
\text { localidade }\end{array}$ \\
\hline \hline $\begin{array}{l}\text { Chamada } \\
\text { de Voz }\end{array}$ & $\$ 0,30 / \mathrm{min}$ & $\$ 0,30 / \mathrm{min}$ & $\$ 0,30 / \mathrm{min}$ & $\$ 0,30 / \mathrm{min}$ \\
\hline $\begin{array}{l}\text { Chamada } \\
\text { de Dados }\end{array}$ & $\$ 0,00 / \mathrm{min}^{*}$ & $\$ 0,00 / \mathrm{min}^{*}$ & $\$ 0,00 / \mathrm{min}^{*}$ & $\$ 0,00 / \mathrm{min}^{*}$ \\
\hline \begin{tabular}{l|c|c|c|} 
SMS \\
torpedos)
\end{tabular} & $\$ 0,30 / \mathrm{msg}$ & $\$ 0,30 / \mathrm{msg}$ & $\$ 0,30 / \mathrm{msg}$ & $\$ 0,30 / \mathrm{msg}$ \\
\hline
\end{tabular}

tal serviço. 
Com relação ao custo de realização de chamadas nas duas redes, observe que no sistema GSM, os custos vão aumentando conforme o usuário vai se distanciando fisicamente da rede de sua operadora, enquanto que as distâncias não tornam as chamadas caras no sistema GAN, fazendo com que sejam obtidos preços para utilização significantemente menores, principalmente considerando o tempo de utilização do serviço e o custo de aquisição da Internet (não incluso no valor da ligação). Neste aspecto, o acesso genérico mostra-se uma alternativa vantajosa em relação ao acesso comum GSM.

\section{CONCLUSÕES}

Após o estudo da utilização do acesso genérico ao núcleo da rede GSM, concluímos que esta tecnologia, que se utiliza de outras redes instaladas e consolidadas como meios de acesso até o núcleo da rede da operadora, interconectando diferentes sistemas ao modelo GSM padrão, é capaz de substituir com sucesso o acesso aéreo comum com o acesso genérico via uma rede mundial como a Internet. Com a GAN, é possível incluir pessoas fora da área da cobertura ao núcleo da rede, sem a necessidade de passagem pelos elementos comuns do acesso GSM. Esta portabilidade no acesso permite que a cobertura seja aumentada e alcance áreas que o sistema GSM não alcança. Esta alternativa de acesso em nada interfere na segurança do sistema como um todo, uma vez que todas as transferências de informações entre o celular e a rede é feito através de um túnel seguro IPSec, garantindo a integridade, a privacidade e a confiança nos dados transportados entre as entidades. A segurança é um dos pontos fortes do sistema, pois também prevê que o celular e a operadora autentiquem-se um ao outro, evitando que elementos maliciosos enganem o sistema fazendo-se passar por um dos dois. Além de oferecer a vantagem de permitir que um usuário utilize os serviços oferecidos pela operadora em qualquer lugar onde exista acesso à Internet, ainda permite uma economia maior nos gastos realizados com as chamadas de voz, provê o poder da portabilidade (como escolher em que AP iniciar sua comunicação com o SEGW), a preservação do núcleo da rede, das conversas e dados trafegados na rede e a capacidade do sistema de utilizar todos os serviços oferecidos pela rede sem a necessidade de configurações extras. $\mathrm{O}$ acesso genérico não interfere diretamente no desempenho do celular dentro da rede, porém, de fato, necessita de mais tempo para tornar-se disponível ao usuário, mesmo apresentando uma utilização de tempo maior em relação ao acesso GSM padrão na requisição de serviços à rede. Ainda existe uma diferença de tempo entre a utilização dos serviços pelos dois sistemas, mas ela é pequena o suficiente para não ser percebida pelo usuário final ou tornarse um empecilho à utilização do sistema. Apesar da existência de outras tecnologias capazes de realizar em parte o mesmo que o acesso genérico, aqui o usuário final e a operadora contam com a convergência a favor da redução de custos de implementação e utilização como também a facilidade na utilização deste meio de comunicação.

Observamos que é necessário um estudo mais aprofundado do impacto desta tecnologia tanto na Internet quanto no núcleo da rede. Diante disto, sugerimos como possíveis trabalhos futuros, a análise de ruído no sinal emitido e recebido, através da Internet, na tecnologia GAN, a análise da implementação de outras formas de criptografia dos dados trafegados entre o celular e o SEGW (como o HMAC-SHA1024), a análise do impacto da retransmissão gerada pelo encapsulamento e desencapsulamento da sinalização GSM pela GAN e a influência de redes de mesmo espectro de sinal não-licenciado, como o Bluetooth e o WiMax, na utilização do sistema de acesso genérico ao núcleo da rede GSM.

\section{AGRADECIMENTOS}

Gostaríamos de primeiramente agradecer a Daniel Cunha e à Maria de Lourdes Alcoforado pelo apoio e ajuda recebida durante a realização deste trabalho. Também aproveitamos para agradecer ao CESAR por haver nos representado perante as operadoras americanas de telefonia celular T-Mobile e AT\&T que nos proporcionou realizar as medidas a partir do Brasil para o caso de acesso genérico e nos forneceram os valores dos custos de ligações para fins de comparação. Finalmente, agradecemos à Fundação de Amparo à Ciência e Tecnologia do Estado de Pernambuco (FACEPE), à Escola Politécnica de Pernambuco, e também ao Conselho Nacional de Desenvolvimento Científico e Tecnológico (CNPq).

\section{REFERÊNCIAS}

[1] ESTRIN, D.; CULlER, D; PISKER, K; SUKHATME, G. Connecting the Physical World with Pervasive Networks. IEEE Pervasive Computing. vol. 01, n. 01, p. 59-69, Jan. 2002.

[2] http://www.gsmworld.com, acesso em 28/06/2008.

[3] DOUSKALIS, B. - Putting VoIP To Work, $1^{\text {st }}$ Ed. Prentice Hall, 2001

[4] MOULY, M.; Pautet, M.B. The GSM System for Mobile Communication, $1^{\text {st }}$ Ed. PALAISEAU: Cell \& Sys, 1992.

[5] GRATTON, D. Developing Practical Wireless Applications, $1^{\text {st }}$ Ed. Digital Press, 2006.

[6] MENDES, D. Redes De Computadores - Teoria e Prática, $1^{\mathrm{a}}$ Ed. Novatec, 2007.

[7] KAARANEN, H.; AHTIAINEN, A.; LAITINEN, L.; NAGHIAN, S.; NIEMI, V. UMTS Networks, $2^{\text {nd }}$ Ed. John Wiley \& Sons, LTD, 2002.

[8] GOODMAN, D. Wireless Personal Communication Systems, $1^{\text {st }}$ Ed. Addison Wesley, 1997.

[9] TANEMBAuM, A. Redes de Computadores, $4^{\mathrm{a}}$ Ed. Campus Editora, LTDA, 2003.

[10] ALCANTARA NETO, P. Telefonia, Recife, 2005. Escola Politécnica de Pernambuco - UPE.

[11] KUROSE, J.; ROSS, K. Redes de Computadores e a Internet: Uma Nova Abordagem, $4^{\mathrm{a}}$ Ed. Makron Books., 2004.

[12] VIKBERG, Jari. Generic access to the A/Gb interface; Stage 2. Third Generation Partnership Project - 3GPP GSM EDGE Radio Access Network - GERAN Meetings, v. 35, no 43.318, 2007. Disponível em: http://www.3gpp.org/ftp/Specs/2007-09/Rel-7/43_series/43318-730.zip, acesso em 02/10/2007.

[13] VIKBERG, Jari. Generic Access (GA) to the A/Gb interface; Mobile GA interface layer 3 specification. Third Generation Partnership Project - 3GPP GSM EDGE Radio Access Network - GERAN Meetings, v. $35, \quad \mathrm{n}^{\circ} \quad 44.318, \quad 2007$. Disponível em: http://www.3gpp.org/ftp/Specs/2007-09/Rel-7/44_series/44318-740.zip, acesso em 02/10/2007. 